\title{
Schweizerisch-deutsche Beziehungen in Medizin und Naturwissenschaft unter besonderer Berücksichtigung des 18. Jahrhunderts
}

\author{
Von G. Rudolph
}

Wissenschaftliche Beziehungen, Verbindungen zwischen den geistigen Strömungen der einzelnen Völker haben von jeher etwas Anziehendes. Man möchte glauben, daß in derartigen Berührungszonen, ähnlich wie bei gewissen pharmakodynamischen Substanzen, sich die Wirkungen verstärken, oder zumindest deutlicher zutage treten.

Für die Beziehung der Schweiz zu ihren Nachbarländern, besonders zum deutschen und zum französischen Sprachraum gibt es zahlreiche Beispiele. Sicher spielt die geographische Situation eine Rolle. Auch der natürliche Vorzug der Mehrsprachigkeit. Von besonderer Bedeutung ist aber wohl die geistige Grundstimmung, die schon früh die Entwicklung eines höheren intellektuellen Niveaus begünstigte. In diesem Sinne läßt sich der von Buess ${ }^{1}$ zitierte, das Basel des 15. Jahrhunderts kennzeichnende Ausspruch des Historikers Rudolf Wackernagel wohl über den Baseler Bereich hinaus ausweiten: «Überall aber und mitten in aller Rauheit und Ruhelosigkeit des Lebens meldet sich ein Verlangen nach höheren geistigen Werten; unter der Last äußerer Ereignisse dringt es um so ungeduldiger hervor.»

Vielleicht kann man bei den wirksam werdenden Einflüssen zwischen Beziehungen unterscheiden, die von Einzelpersönlichkeiten geknüpft, getragen, überpflanzt werden und Ausstrahlungen, die im Lande fixiert bleiben, aber so mächtig sind, daß sie in weitem Umkreis die Blicke auf den Focus ziehen. So hat MANI ${ }^{2}$ Basel als ein Zentrum des medizinischen Humanismus im 16. Jahrhundert geschildert, das in der Pflege antiker Überlieferung neben Venedig, Paris und Lyon trat. Aber es war auch ein Zentrum anatomischer und botanischer Forschung, deren Ausbreitung wiederum durch

1 H. Buess, Streiflichter auf die Geschichte der Medizinischen Fakultät Basel, Verh. XIX. int. Kongr. Geschichte der Medizin, Basel 1964, p. 396-401 (Karger, Basel/New York 1966).

2 N.MANI, Basel als Zentrum der antiken medizinischen Überlieferung im 16. Jahrhundert, Verh. XIX. int. Kongr. Geschichte der Medizin, Basel 1964, p. 395 (Karger, Basel/ New York 1966). 
die hohen technischen Leistungen der Buchdrucker am Oberrhein begünstigt wurde. Eduard Fueter ${ }^{3}$ hat in einer Übersicht auf die Wirkung der Zürcher naturforschenden Ärzte hingewiesen. - Die von der Einzelpersönlichkeit geknüpften Beziehungen können vom kurzen oder längeren Studienaufenthalt im Ausland bis zur vorübergehenden oder dauernden Einfügung in den fremden Lebensbereich gehen. Die Ausdehnung wissenschaftlicher Korrespondenz oder die Heranziehung ausländischer Schüler ermöglichen auch den Seßhaften weitreichende Kontakte.

Eine planmäßige Förderung des wissenschaftlichen Austausches mit dem In- und Auslande wurde im 17. Jahrhundert durch die Einrichtung wissenschaftlicher Gesellschaften eingeleitet. Da findet sich deutscherseits neben ephemeren Gründungen (Jungrus 1622) - zehn Jahre älter als die Royal Society - die Schweinfurter Academia Naturae Curiosorum (1652), die heute noch als Leopoldina-Carolina (seit 1878 mit ständigem Sitz in Halle)

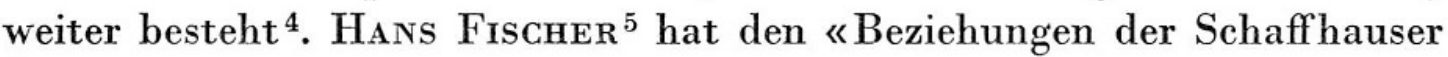
Ärzteschule des 17.Jahrhunderts zur Deutschen Akademie der Naturforscher» eine wichtige Studie gewidmet. Johann Conrad Brunner (1653 bis 1727) seit 1686 o. Prof. in Heidelberg, ist zugleich ein Beispiel für das Wirken schweizerischer Gelehrter in Deutschland und die zumindest im 17. Jahrhundert nicht immer angenehme Situation des Hochschullehrers, wie Edith Küthmann (1957) im Gesnerus dargestellt hat ${ }^{6}$.

Die Gemeinsamkeit der deutschen Sprache spielte für die Gelehrten zunächst wohl keine, oder nur eine sehr untergeordnete Rolle, obwohl schon im 16. Jahrhundert Schriften vonVAdian (JoAChim von WatT 1484-1551) und von Paragelsus (1493-1541) in deutscher Sprache erschienen sind?

3 E. Fueter, Zürcher Ärzte als Naturforscher, Verh. XIX. int. Kongr. Geschichte der Medizin, Basel 1964, 402-408 (Karger, Basel/New York 1966).

${ }^{4}$ Vgl. S.F.Mason, Geschichte der Naturwissenschaft in der Entwicklung ihrer Denkweisen, deutsche Ausgabe unter Mitwirkung van K.M.MeYer-Aвich besorgt von B.STICKER, Kröner, Stuttgart 1961.

5 H.Fischer, Beziehungen der Schaffhauser Ärzteschule des 17. Jahrhunderts zur Deutschen Akademie der Naturforscher, Festgabe zur 147. Jahresversammlung der Schweizerischen Naturforschenden Gesellschaft 29.September bis 1.Oktober 1967 in Schaffhausen, 28 p., aus Mitteilungen der Naturforschenden Gesellschaft Schaffhausen, Band XXVIII, Jg. 1963/67.

${ }^{6}$ Edith Küthmann, Johann Conrad Brunner in Heidelberg als Hochschullehrer und Therapeut, Gesnerus 14 (1957) 119-140.

7 Vgl. H.Buess, Schweizer Ärzte als Forscher, Entdecker und Erfinder, herausgegeben von der Ciba Aktiengesellschaft, Basel 1946. 
Das ändert sich freilich in dem Maße als die Vulgärsprache in wissenschaftlichen Veröffentlichungen sich neben das Latein schiebt und schließlich an dessen Stelle tritt. Das Französische, das zweifellos im schweizerischdeutschen Austausch auch eine Rolle gespielt hat, ist nur für verhältnismäßig kurze Zeit als «langue universelle ${ }^{8}$ wie in der Berliner Akademiesprache von Bedeutung gewesen.

Im 19. Jahrhundert entwickelt sich schließlich in weitem Umfang das deutsche medizinische Zeitschriftenwesen, und es ist naheliegend - allein schon wegen des Verbreitungsgebiets dieser Zeitschriften -, daß deutschsprachige schweizerische Autoren in zunehmendem Maße in Deutschland publizieren. Trotz der bedeutenden verlegerischen Tradition in der Schweiz erscheinen im Laufe des 19. Jahrhunderts zunehmend wichtige Monographien in Deutschland bis zu den Publikationen etwa von Eugen BLeuLer (1857-1939), von Otтo Naegeli (1871-1938) und darüber hinaus?. Daß etwa Gabriel Gustav Valentin (1810-1883) seinen Grundriß der Physiologie (1847) bei Vieweg in Braunschweig herausbrachte oder Moritz Schiff (1823-1896) seine Muskel- und Nervenphysiologie (1858-1859) bei Schauenburg in Lahr, könnte noch als alte Anhänglichkeit an ihr Ursprungsland gewertet werden ${ }^{9}$.

Einen relativen Aufschluß über die Bedeutung, die wissenschaftlichen Autoren in ihrer Zeit zugemessen wurde, könnte auch in der Zahl der Buchveröffentlichungen enthalten sein, die von den großen Bibliotheken angeschafft wurden. So enthält die medizinische Abteilung der Kieler Universitätsbibliothek, die im 17. Jahrhundert gegründet und sicher nie glänzend dotiert war, in ihren nahezu lückenlos über den Krieg geretteten Beständen zahlreiche Titel bzw. Ausgaben bekannter schweizerischer Autoren (seien diese nun in der Schweiz oder in anderen Ländern erschienen), wie Théophil Bonet (1620-1689) [8 Buchtitel], Johann von Muralt (1645-1733) [5], Harder (1656-1711) [4], Wepfer (1620-1695) [5], Brunner [4], Peyer (1653-1712) [3]. Das gleiche gilt im 18. Jahrhundert für Haller oder für GirTanNer, auf den noch zurückzukommen sein wird.

Solche Daten verlocken natürlich, die Einfluß- oder Austauschrate zu quantifizieren. Das ist meines Wissens bisher noch nicht oder nicht syste-

${ }^{8}$ Siehe auch Antoine de Rivarol, Discours sur les causes de l'universalité de la langue française, Preisschrift der Berliner Akademie 1784.

9 Vgl. K. E. Rотнsснuн, Geschichte der Physiologie, Springer, Berlin/Göttingen/Heidelberg 1953. 
matisch versucht worden, obwohl statistische Elemente in der Betrachtung wissenschaftlicher Beziehungen zwischen den Nationen schon recht glücklich von Alphons de Candolle (1806-1893) verwandt sind ${ }^{10}$ und zu einer Art géographie raisonnée des scientifiques et de leur influence hätten führen können.

Totz dieser sicher lohnenden Perspektive möchte ich, mehr herkömmlich, an einigen Einzelpersönlichkeiten auf ihre Zeitwirkung hinweisen und dabei eine ganz umschriebene Epoche herausstellen. Denn ohne Zweifel könnte man sehr lange über schweizerisch-deutsche Wissenschaftsbeziehungen sprechen, ohne den Gegenstand auch nur annähernd zu erschöpfen.

Das 18. Jahrhundert erscheint in mehrfacher Hinsicht bemerkenswert. Nicht nur, daß das philosophische Zeitalter durch systematische Entwicklung der Kritik und die Anwendung der Grundsätze Bacons (1561-1626) ${ }^{11}$ den Wissenschaftspositivismus des 19. Jahrhunderts vorbereitet, es hat auch als ein ausgesprochen kosmopolitisches Zeitalter und ein Zeitalter allgemeiner geistiger Erweckung den Austausch und die Aufnahme neuer Ideen in besonderem Maße angeregt. Hinzu kam noch der kühne Durchbruch enger Fachbeschränkungen, der nicht nur den Enzyklopädismus eines Conrad Gessner (1516-1565) ${ }^{12}$ weiterentwickelte, am spürbarsten in A. von HALLER (1708-1777), sondern dazu führte, daß namhafte Wissenschaftler oder ärztliche Praktiker wie Haller und Zimmermann (1728-1795) durch ihre Dichtungen und philosophische Schriften eine weitaus stärkere Beachtung in der Öffentlichkeit fanden und dank einer emsig weiterwirkenden Literaturwissenschaft auch behielten.

Der schweizerische Einfluß war gerade im 18. Jahrhundert außerordentlich groß und erstreckte sich auf weite Bereiche der Wissenschaft und der Ästhetik. Die Schriften der Zürcher Bodmer (1698-1783) und Breitinger

10 A. de Candolle, Histoire des Sciences et des Savants depuis deux siècles suivie d'autres études sur des sujets scientifiques en particulier sur la sélection dans l'espèce humaine, Georg, Genève/Bâle/Lyon 1873.

11 Advancement of Learning (1605), Nocum Organum (1620). Vgl. D'Alembert, Discours préliminaire de l'encyclopédie, 1751; siehe auch L.Premuda, Kritische Betrachtungen über den Inhalt der Vorreden zur «Fabrica» von Vesal und zu den «Elementa Physiologiae» von HaLler, Jahrbuch der Universität Düsseldorf 1969/70, p. 353-363.

12 H.Fischer, Conrad Geßner (26. März 1516 bis 13.Dezember 1565), Leben und Werk, Veröffentlichung der Naturforschenden Gesellschaft in Zürich, 1966. 
(1701-1776) um 1740 führten zu einem neuen ästhetischen Bewußtsein ${ }^{13}$. Joh. Georg Sulzer (1720-1779) aus Winterthur, «der seine Stellung an der Berliner Akademie wie eine Art Gesandschaftsposten der Zürcher Kunstrichter im Deutschen Reiche auffaßte» - so der Literarhistoriker Max Koch $1907^{14}$ - hat 1771 in seiner Theorie der schönen Künste BREITINGERS Grundsätze systematisch verarbeitet. Als Erforscher der Empfindung hat Sulzer aber auch in der medizinischen Wissenschaft seinen Platz.

Die in Deutschland lebenden schweizerischen Gelehrten lassen sich einteilen in solche, die an einer Universität lehren und solche, die außeruniversitär ihre Wirksamkeit entfalten. Die außer-universitären sind häufig von Fürsten zu besonderen Aufgaben verpflichtet oder nehmen aktiv teil an der Arbeit wissenschaftlicher Akademien.

Als Universitätslehrer und Forscher, aber auch auf dichterischem Gebiet als Erwecker eines neuen Naturgefühls und Vorbereiter der Klassik - eine deutsche Wiederholung des «enfin Malherbe vint» - die für einen einseitigen kulturhistorischen Aspekt immer noch ausschlaggebend ist - nimmt Albreght von Haller einen hervorragenden Platz ein. Sein Einfluß ist fast unabsehbar und welche Bedeutung ihm allein als Kritiker «für die gesamte Geistesgeschichte der Epoche ${ }^{15}$ zugemessen wird, kommt in den Worten von J.G.Herder (1744-1803) zum Ausdruck: Haller «hat auch als Prosaist so viel verdient um den besseren Geschmack im Vortrage der Wissenschaften, daß ihm auch die deutsche Kritik den ersten Kranz rei-

13 Vgl. G. G. Gervinus, Historische Schriften, 5.Band, Geschichte der Deutschen Dichtung $I V$, Engelmann, Leipzig 1840. Über den Zürcher literarischen Verein: A. KoвERsteIN, Geschichte der Deutschen Nationalliteratur vom zweiten Viertel des achtzehnten Jahrhunderts bis zu Goethes Tod, 5. Auflage von KarL Bartsch, 1.Theil F.C. W. Vogel, Leipzig 1872, p. 47: «Damals standen hinter Zürich andere Städte, die später die bedeutendsten in unserem Literaturleben wurden, noch weit zurück in der Bildung ...»

${ }^{14}$ M. Косн, Geschichte der Deutschen Literatur, 2. Band: Vom 17. Jahrhundert bis zur Gegenwart, Bibliographisches Institut, Leipzig/Wien 1907. - Neben Sulzer sind als Akademiemitglieder in der Klasse der spekulativen Philosophie die Schweizer Nicolas de Béguelin (1714-1789) und Johann Bernhard Merian (1723-1807) zu nennen. Letzter war nach dem Tod von SAMuel Formey (1711-1797) ständiger Sekretär der Akademie, "für die allein er gelebt und gewirkt hat» (J.E.ErdmanN, Grundriß der Geschichte der Philosophie, Berlin 1866).

${ }^{15}$ H. Bloesch zit. nach K.S. GuthKe, Haller und die Literatur (Arbeiten aus der Niedersächsischen Staats- und Landesbibliothek Göttingen, Band 4), Vandenhoeck \& Ruprecht, Göttingen 1962. 
chet. Mitten unter stürmischen Faktionen brachte er ein schmales Blatt deutscher Kritik unter den Schutz einer Sozietät der Wissenschaften selbst und gründete ihm dadurch nicht nur Unparteilichkeit, Billigkeit und Gleichmut, sondern auch Teilnahme am Fortgange des menschlichen Geistes in allen Weltgegenden und Sprachen. Seitdem sind die Göttingischen Gelehrten Anzeigen nicht nur Annalen, sondern auch Befördrinnen und, ohne ein Tribunal zu sein konsularische Fasten und Hülfsquellen der Wissenschaft worden, zu denen man, wenn manche einseitige Kritik verstummt ist, wie durch Lybische Wüsten zum stillen Kenntnis gebenden Orakel der Wissenschaft reiset und dabei immer noch Hallers und seiner Nachfolger Namen segnet». - So in den Briefen «zur Beförderung der Humanität» ${ }^{16}$, in denen Herder die Bilanz seines Jahrhunderts zog.

Haller hat sich in die ästhetischen Fehden seiner Zeitgenossen (so die Bodmer-GotTsched-Kontroverse) nicht willentlich eingeschaltet, und noch 1772 schrieb er an Christoph Gottlieb von Murr (1733-1811) ${ }^{17}$ : «Überhaupt bedaure ich allemal die Wissenschaften und die Gelehrten, wenn sie in Streitigkeiten gerathen und ein oft unwissendes und unachtsames Publikum zu Richtern über sich aufwerfen, sie die billig selber als Richter sitzen sollen.» Das hinderte ihn natürlich nicht, in naturwissenschaftlichen Fragen entschiedene Positionen zu beziehen, so daß Goethe (1749-1832) über den «Bildungstrieb» (1820) schreiben konnte: «Hier fand ich nun meinen Kaspar Friedrich WolfF ${ }^{18}$ als Mittelglied zwischen Haller und BonNET $^{19}$ auf der einen und BLumenbach ${ }^{20}$ auf der anderen Seite.» ${ }^{21}$

Haller selbst, der seine wissenschaftlichen Arbeiten lateinisch schrieb und herausgab, wurde zu seinen Lebzeiten immer wieder ins Deutsche über-

16 Zit. nach Guthke, p. 24.

17 Brief vom 26. November 1772. In einem vorausgegangenen Schreiben (26. September 1772) heißt es: «Mir ist indessen ... leid, daß die Gelehrten sich wie Gladiatoren der Welt zum Schauspiele geben und dadurch in Stellungen gesetzt werden, daß eine jede Zeitung ihr Gemüth erschüttert und Urteile für sie wichtig werden, die sie sonst verachten würden», aus L. Hrnzex, Albrecht von Hallers Gedichte (Einleitung, p. CDLXXXIX), Huber, Frauenfeld 1882.

18 Caspar Friedrich Wolff aus Berlin (1735-1794), Epigenetiker.

19 Charles Bonnet aus Genf (1720-1793), Palingenetiker.

20 Johann Friedrich Blumenbach aus Gotha (1752-1840), Über den Bildungstrieb und das Zeugungsgeschäft, Göttingen 1781.

21 J.W.v.GoEthe, Schriften zur Naturwissenschaft I, in Sämtliche Werke, Jubiläumsausgabe, Band 39, Cotta, Stuttgart/Berlin o.J. 
setzt und damit einem großen, humanistisch weniger gebildeten Leserkreis bekannt ${ }^{22}$.

Länger als Haller in Göttingen (1736-1753) hat Leonhard Euler (1707-1783) in Berlin gewirkt (1741-1766). «EULER, einer von denjenigen Männern, die bestimmt sind, wieder von vorn anzufangen, wenn sie auch in eine noch so reiche Ernte ihrer Vorgänger geraten», wie GoETHE ${ }^{23}$ ihn charakterisiert hat, hatte eine bedeutende Tätigkeit an der Berliner Akademie entfaltet. Seine «Lettres à une princesse d'Allemagne sur divers sujèts de physique et de philosophie», für die Nichte Friedrichs II., die Prinzessin von Anhalt-Dessau geschrieben, hatten als Werk der späteren Aufklärung einen bedeutenden Einfluß ${ }^{24}$. «Le nom d'Euler, si grand dans les sciences, l'idée imposante que l'on se forme de ses ouvrages, destinés à

22 Das betrifft auch die von Haller herausgegebenen wissenschaftlichen Abhandlungen, so beispielsweise Herrn Albrecht von Hallers Sammlung academischer Streitschriften die Geschichte und Heilung der Krankheiten betreffend (3 Bände, Joh. Heinr. Kühnlin, Helmstedt 1779-1780), deren zweiten Band der Übersetzer und Kommentator LoRENz CreLL (1744-1816) Professor in Helmstedt bis 1809 (später in Göttingen) «der KöniglichGroßbritannischen Gesellschaft der Wissenschaften zu Göttingen widmet». Er schreibt in der Vorrede: «So groß der entschiedene Werth der Sammlung practischer Streitschriften vom Herrn v. Haller ist; so sehr wäre es zu wünschen, daß sie viel allgemeiner in den Händen, besonders junger Ärzte, seyn mögte, als man sie würklich bey ihnen antrifft. Die Ursach hievon läßt sich, bey dem besten Willen vieler Ärzte, aus der Seltenheit dieser Sammlung ... und aus ihrem hohen Preise, leicht begreifen. Beyden Ursachen abzuhelfen, und dadurch die weitere Ausbreitung (im Original gesperrt) so mancher nützlichen Wahrheit zu befördern, entschloß ich mich zu gegenwärtiger Arbeit. »- Eine wohl nahezu vollständige Übersicht ergibt sich aus Susanne LundSGAARD-HANSEN-voN Fischer, Verzeichnis der gedruckten Schriften Albrecht von Hallers, Berner Beiträge zur Geschichte der Medizin und der Naturwissenschaften, Nr. 18), Haupt, Bern 1959.

${ }^{23}$ J.W.v. Goethe, Materialien zur Geschichte der Farbenlehre, Schriften zur Naturwissenschaft II in Sämtliche Werke, Jubiläumsausgabe, Band 40, Cotta, Stuttgart/Berlin o. J.

${ }^{24}$ Geschrieben von 1760 bis 1762 wurden die Briefe zuerst 1768 bis 1772 (in 3 Bänden) in St.Petersburg veröffentlicht. Es folgte 1787-1789 die Ausgabe von Condorcet. Emile SaIsset, der die fünfte Ausgabe (nach LaBey 1812 und Cournot 1842) veranstaltet hat, charakterisiert das Werk aus der Sicht des Metaphysikers in der Nachfolge der Restauration (Introduction p. V): «Les lettres ... nous présentent le spectacle animé de ce temps de crise, d'épuisement et de dissolution. Euler s'y montre l'ennemi déclaré des Wolffiens... Il combat avec force, avec passion la monadologie et l'harmonie préétablie... auxquelles il n'épargne pas même des sarcasmes peu dignes d'un esprit si grave. Du reste, Euler ne prétend pas substituer un nouveau système à celui de Leibnitz. Occupé d'autres objets, dominé d'ailleurs par l'esprit de son temps, il se défie des systèmes.» 
développer ce que l'analyse a de plus épineux et de plus abstrait - schreibt der Mathematiker Condorcet (1743-1794) ${ }^{25}$ - donnent à ces lettres si simples, si faciles, un charme singulier: - und hier das Geheimnis ihres Erfolges - ceux qui n'ont pas étudié les mathematiques, flattés peut-être de pouvoir entendre un ouvrage d'Euler, lui savent gré de s'être mis à leur portée...»

Ein Schweizer aus Chur, der bis zum Generalchirurg der friderizianischen Armee avancierte, war JohanN Ulrich v. Bilguer (1720-1796 ${ }^{26}$. Er hat sich um die Hebung des Ausbildungsstandes der Wundärzte verdient gemacht, die nach den Äußerungen von ERnst-Gottrried Baldinger (1738 bis 1804) lamentabel gewesen sein mußte ${ }^{27}$. Schuld war das geringe wissenschaftliche Interesse der Ärzte, namentlich die unter den Feldärzten verbreitete «Bibliophobie». Hatten einige doch kein weiteres Buch bei sich als den Tod Abels von S.Gessner (so Baldinger) ${ }^{28}$. Bemerkenswerterweise ist selbst dies einzige Buch schweizerischen Ursprungs. - BILGuER ist der Verfasser bedeutender militärchirurgischer Schriften. Seine zunächst lateinisch erschienene Dissertation über den Mißbrauch der GliedmaßenAmputationen wurde von Tissot ${ }^{29} 1764$ ins Französische übersetzt und erfuhr dann noch weitere Übersetzungen in das Holländische und Spanische.

Auf einen wichtigen Beitrag zur Naturforschung des 18. Jahrhunderts, den merkwürdigerweise Roтнsснuн in seiner Darstellung der prägalvanischen Elektrizität ${ }^{30}$ nicht erwähnt, hat Emil du Bors-Reymond (1818 bis 1896) aufmerksam gemacht. Er sagt in seiner Rede über das Nationalgefühl

25 Marie-Jean-Antoine-Nicolas Caritat de Condorcet, Eloge d'Euler, in Lettres d'Euler à une princesse d'Allemagne..., avec une introduction et des notes par EMILE SAIsset, 2 t., Charpentier, Paris 1859.

26 Bibliographie von A. J.L. Jourdan in Biographie médicale, t. 2, p. 256-258, Panckoucke, Paris 1820.

27 Vgl. G.L.MAмLоск, Friedrichs des Großen Korrespondenz mit Ärzten, Enke, Stuttgart 1907.

${ }^{28}$ Salomon Gessner aus Zürich (1730-1788). Seine Schriften waren in zahlreichen deutschen Nachdrucken (z.B. Fleischhauer, Reutlingen) verbreitet.

29 Samuel Auguste André David Tissot aus Lausanne (1728-1797). Hinweis auf seine Übersetzertätigkeit in H.W.Bucher, Tissot und sein Traité des nerfs, ein Beitrag zur Medizingeschichte der schweizerischen Aufklärung, p. 59, Zürcher medizinigeschichtliche Abhandlungen, Neue Reihe I, Zürich 1958.

${ }^{30}$ K. E. Rотнsснuн, Aus der Frühzeit der Elektrobiologie, Elektromedizin 4. (1959) 201-217, Zur Geschichte der physiologischen Reizmethodik im 17. und 18. Jahrhundert, Gesnerus 23 (1966) 147-160, ebenso Die Anfänge der Elektrophysiologie, Bild der Wissenschaft 1966, 106-113, siehe auch Anmerkung 9. 
$(1878)^{31}$ : «Welches Gewicht hätte man nicht anderswo der bei uns ganz unbeachteten Tatsache beigelegt, daß die erste galvanische Erscheinung, welche noch Volta den Schlüssel zu Calvanis Versuchen gab, hier in Berlin, von einem unserer Vorgänger beobachtet wurde.» Es handelt sich um Sulzers Versuch über den elektrischen Geschmack aus dem Jahre 1752. Du Bors weist wiederholt in seinen «Reden» auf diese grundlegende Entdeckung Sulzers hin, teilt aber nicht den Vornamen des Autors mit. Die ausführlichere Beschreibung in seiner tierischen Elektrizität ${ }^{32}$ läßt aber keinen Zweifel darüber, daß es sich um den Mathematiker und Philosophen sowie späteren Akademiedirektor (1776) Johann Georg SUlzer handelt ${ }^{33}$.

$\mathrm{Zu}$ dem friderizianischen Strahlenkranz der Euler, Sulzer und BiLGUER, dem der König auch gerne HALLER zugesellt hätte ${ }^{27}$, ist in gewisser Weise auch Johann Georg Zimmermann (1728-1795) aus Brugg zu rechnen, «einer der bedeutendsten Praktiker und gesuchtesten Ärzte des 18. Jahrhunderts ${ }^{27}$.

«Il y a huit mois que je suis fortement attaqué de l'Astme. Les médecins de ce pays-ci Me donnent toutes sortes de drogues, mais qui, plutôt de me procurer du soulagement ne font qu'empirer le mal. La réputation de votre habileté étant étendue dans tout les nord de l'Europe, je serais bien aise, si vous vouliez faire un tour pour quinze jours dans ce pays-ci, pour vous consulter sur l'état de ma santé et ses circonstances» schrieb Friedrich 1786 an Zimmermann. Dieser antwortete: «Si on était bon médecin à proportion du désir de l'être, je crois que Votre Majesté serait guérie au premier instant où j'aurai l'honneur de la voir. - J'attends cet instant avec impatience, enthousiasme et courage $\gg .^{34}$

31 E. DU Bors-Reymond, Reden, in zwei Bänden, zweite vervollständigte Ausgabe, herausgegeben von Estelle du Bors-Reymond, Veit \& Comp., Leipzig 1912.

32 Untersuchungen über thierische Elektrizität, Band I, Berlin 1848, S. 54 Anm.

33 Über Johann Caspar Sulzer (geboren 1716 zu Winterthur, gestorben 1799 zu Gotha) und seinen Sohn Friedrich Gabriel Sulzer (geboren 1749 zu Gotha, gestorben 1830 zu Altenburg), «zwei hervorragende Mediziner schweizerischer Herkunft im goethezeitlichen Thüringen» hat H.Petzsch berichtet (Verh. XIX. int. Kongr. Geschichte der Medizin, Basel 1964, p. 416-419, Karger, Basel/New York 1966): «Häufig werden sowohl Johann Caspar als auch Friedrich Gabriel Sulzer fälschlicherweise mit ihrem entfernten Verwandten identifiziert und verwechselt, dem ebenfalls aus Winterthur stammenden Polyhistor und Ästhetiker Johann Georg Sulzer (1720-1779), der in Berlin wirkte und lebte» (p. 419). Nach Paulus (?) Usteri (1788-1831), Arzt und Bibliothekar in Zürich, 1802 schweizerischer Mitkonsul in Paris, ist dieser der jüngere Bruder des Mediziners Johann Caspar (Biographie universelle 44 (Paris 1826) 212.

34 Siehe Mamlock (Anm. 27) Briefe 169 und 170, p. 154-156. 
Zimmermanns philosophische Schriften, besonders über die Einsamkeit ${ }^{35}$ - wie Haeser ${ }^{36}$ etwas boshaft bemerkt «die Frucht von Zimmermanns 14jährigem Still-Leben in Brugg» - haben seinen Namen in Deutschland bekannter gemacht als seine verdienstvollen Arbeiten über die Erfahrung in der Arzneykunst (Zürich 1763) ${ }^{37}$, über die Ruhr (Zürich 1767) ${ }^{38}$ oder die ebenfalls im Sinne der Aufklärung wirkende kleine Schrift von der WindEpidemie in Hannover (1772) ${ }^{39}$. In seinem dem Ratsherrn Schмidт zu Brugg vom 27.0ktober 1771 gegebenen Bericht ${ }^{40}$ über seinen Besuch in Potsdam, der ungemein lebhaft seine Unterhaltung mit Friedrich II. schildert, schreibt er: «Unaussprechlich freut es mich, zu sehen, wie viel itzt die Schweizer bey dem Könige gelten. Er hat gesagt, daß er keine anderen Physicos und Mathematicos bey der Akademie haben wolle als Schweizer.» ${ }^{41}$ Die von kleineren Zeitgenossen dem weltmännischen Arzt vorgeworfene Eitelkeit und Selbstgefälligkeit, die weder Goethe noch Friedrich den Großen ${ }^{27}$ gestört hat, hat Zimmermanns Nachbild in der Geschichte verdunkelt. Die Erfahrung in der Arzneykunst, die sich 1782 der emigrierte junge SchiLler (1759-1805) als dringlichste Lektüre gewünscht hatte ${ }^{42}$, ist im 19. Jahrhundert ohne Verständnis beurteilt worden. AGKerknecht hat

35 Von der Einsamkeit (1756) erweitert zu Betrachtungen über die Einsamkeit, 4 Bände 1784.

${ }^{36}$ H.HAeser, Lehrbuch der Geschichte der Medizin, dritte Bearbeitung, 2. Band, Fischer, Jena 1881.

37 Von der Erfahrung in der Arzneykunst, zwei Teile, Heidegger und Compagnie, Zürich 1763 und 1764 .

38 Von der Ruhr unter dem Volke im Jahre 1765 und denen mit derselben eingedrungenen Vorurtheilen, nebst einigen allgemeinen Aussichten in die Heilung dieser Vorurtheile, Fueßlin und Compagnie, Zürich 1767.

39 Von der Wind-Epidemie in der Stadt Hannover und der sogenannten Neuen Krankheit aus dem Hannoverschen Magazin, (5) den 17. Januar und (6) den 20. Januar 1772, erneut abgedruckt in Wilhelm Heinrich Sebastian Bucholz [1734-1798], Nachricht von dem jetzt herrschenden Fleck- und Friesel-Fieber, Hoffmann, Weimar 1772 (p. 49-88) mit der Anmerkung: «Einige satyrische Züge, welche Herr Leib-Arzt ZimmermanN im Anfange seines Aufsatzes miteingewebt, und welche doch des schönen Styls wegen, lesenswerth sind, habe ich mit abdrucken zu lassen, mich nicht entbrechen können.»

40 Johann Georg Zimmermanns Briefe an einige seiner Freunde in der Schweiz, herausgegeben von Albrecht Rengger, Sauerländer, Aarau 1830.

41 Ibid., p. 153.

42 Schillers Briefe, herausgegeben und mit Anmerkungen versehen von Fritz Jonas, Kritische Gesamtausgabe, 1. Band, Deutsche Verlags-Anstalt, Stuttgart/Leipzig/Berlin/ Wien o.J., Brief 45, An Reinwald, p. 85-86. In der Aufstellung werden auch die philosophischen Schriften von Sulzer genannt. 
dankenswerterweise in seiner Geschichte der Therapie auf dieses für die medizinische Aufklärung so wichtige Werk wieder aufmerksam gemacht ${ }^{43}$.

Neben den Sternen 1. und 2. Ordnung sind auch solche minderen Glanzes, aber für die Erforschung des medizinischen Denkens im 18. Jahrhundert gleichwohl von Interesse wie der Arzt und Mathematiker Јон. ЈАк. Ritter (1714-1750) aus Bern ${ }^{44}$, dessen Baseler Dissertation ${ }^{45}$ Haller in seine Disputationes ad morborum historiam (Lausanne 1757-1760) aufgenommen hat.

Die Reihe schweizerischer Gelehrter im Deutschland des 18. Jahrhunderts möchte ich mit Christoph Girtanner (1760-1800) aus St.Gallen beschließen. Das Motto aus Cicero, das er dem 3.Band seiner Abhandlung über die venerische Krankheit (2.Auflage, Göttingen 1793) vorangestellt hat, könnte als Leitgedanke aufklärerischen Kritizismus und Rationalismus beinahe über allen kritischen Schriften der Epoche stehen: «Opinionum commenta delet dies, naturae judicia confirmat.»

Das Göttingen des ausklingenden 18. Jahrhunderts, in dem Girtanner als Arzt, Forscher und überaus fruchtbarer Schriftsteller - freilich ohne universitäre Bindung - wirkte, hat der Göttinger Anglist Herbert SchöfFLER in seiner Studie über Lichtenberg (1742-1799) ${ }^{46}$ geschildert. LichTENBERG hatte am physiognomischen Feuer Lavaters (1741-1801) seinen geistvollen Widerspruch entzündet ${ }^{47}$. Er hat auch Girtanner, über den nur ImMANuel KaNt (1724-1804) uneingeschränktes Lob sprach ${ }^{48}$, kritisiert, und zwar dort, wo seine bleibenden Verdienste liegen, in der Kritik

43 E. H. Aскевкnecht, Therapie von den Primitiven bis zum 20. Jahrhundert, Enke, Stuttgart 1970, p. 26-27, 92-93.

44 Ritter, der in der anatomischen Lehre hervorgetreten war und 1747 eine Professur an der Universität Franeker bekleidete, war vorübergehend Leibarzt des Landgrafen von Hessen-Homburg und starb in Ober-Peilau bei Nimptsch in Schlesien (vgl. Biographie médicale 7, p. 27-28, Panckoucke, Paris 1825).

45 De impossibilitate et possibilitate abstinentiae longae, a cibo et potu ..., Basil. 1737.

${ }^{46}$ H. SchöfFLER, Lichtenberg (Worte Göttinger Gedenkens, gesprochen vor der Universität zum 200. Geburtstage Lichtenbergs), Dieterichsche Verlagsbuchhandlung, Leipzig 1943.

47 Vgl. Lavater über den «Witzler Lichtenberg» an HamanN (1730-1788) in JohanN Georg Hamann, Briefwechsel, herausgegeben von Arthur Henkel, 4. Band, 1778-1782, Insel, Wiesbaden 1959, Brief 524, p. 97-98. Eine ausführliche Darstellung der Kontroverse findet sich bei G. G. Gervinus (siehe Anm. 13) 6.Band, V; p. 290 f.

48 Der Anthropologie II. Teil (Einteilung der Physiognomik D) in Vermischte Schriften von Immanuel Kant, p. 522, Band 1 der Sämtlichen Werke, Inselverlag, Leipzig 1912. 
der Phlogiston -Theorie. In seinen Aphorismen über Chemie ${ }^{49}$ bezieht er sich direkt darauf: «Der abscheulichste Satz des antiphlogistischen JacobinerClubs ist wohl dieser, daß es flüssige Körper in der Natur gibt, daran ist der Druck der Atmosphäre ganz allein schuld. Ohne den Druck der Atmosphäre würden alle Körper entweder im festen, oder im luftförmigen Zustand seyn. Dieses sind Girtanners Worte.» Und einige Seiten weiter ${ }^{50}$ : «Was mich eigentlich bewogen hat, so lange mit meinem Beyfall für die antiphlogistische Chemie zurückzuhalten, ist (verzeihe mir meine schwere Sünde), bloos der enthusiastische Beyfall gewesen, womit sie von einigen Leuten beehrt worden ist, deren Flüchtigkeit im Schließen, Seichtigkeit und Ignoranz in der Naturlehre mir bekannt war.»

Dieser zweite Satz, wenn er wirklich auf GirTanner gemünzt wäre, würde ganz in das von August Hirsch ${ }^{51}$ entworfene Bild hineinpassen. Der angebliche Plagiator ${ }^{52}$ JoHN Browns (1735-1788), der wohl zu seiner eigenen Rechtfertigung später (1793) den Satz Senecas angeführt hat: «Etiam si omnia a veteribus inventa sunt: hoc semper novum erit, usus, et inventorum ab aliis scientia et dispositio ", hat durch CARL WegeLin im Gesnerus ${ }^{53}$ eine späte Ehrenrettung erfahren. Im gleichen Jahr weist Everetr MeNDelsohn in seiner Studie Heat and Life ${ }^{54}$ auf Grrtanners Bedeutung für

49 G. C.Lichtenbergs Physikalische und mathematische Schriften, nach dessen Tode gesammelt und herausgegeben von Ludwig Christian Lichtenberg und Friedrich Kries, 4. Band, p. 194, Dietrich, Göttingen 1806.

50 Ibid., p. 199.

51 A. Hirsch, Artikel Girtanner in Biographisches Lexikon der hervorragenden Ärzte aller Zeiten und Völker, Wien/Leipzig 1885, Band 2, p. 568-569.

${ }^{52}$ Einen Hinweis auf die Kritik an Girtanner, besonders in bezug auf seine Ausführliche Darstellung des Brownischen Systems der praktischen Heilkunde... (2 Bände, Rosenbusch, Göttingen 1797 und 1798) bzw. die voraufgegangenen «Mémoires sur l'irritabilité considérée comme principe de vie dans la nature organisée » (Journal de Physique 1790) gibt die polemische Stellungnahme von François-Pierre Chaumeton (1775-1819). Dazu auch A.-J.L. Jourdan in Biographie médicale, 4, p. 431-433.

53 C. Wegelin, Dr.. med Christoph Girtanner (1760-1800), Gesnerus 14 (1957) 141-168.

54 E.Mendelsohn, Heat and Life, The development of the Theory of Animal heat, Harvard University Press, Cambridge, Massachusetts 1964, p. 169-171. "The two elements in Girtanner's proposal which were important for the theory of animal heat, that oxygen is absorbed by the blood and that the heat is released from the blood during circulation, were not originated by him; one had been conjectured by Lavoisier and the other contained in Crawfors'd theory. However, bringing the two proposals together in the manner he did foreshadowed an important stream of new research into the site of combustion in the animal body. Even Girtanner's crude beginnings indicated that much could be learned from the analysis of the gases found in the blood» (p. 171). 
die Theorie der Wärmebildung im Organismus hin. Daß der BlumenbachSchüler Girtanner als wichtiger Vorläufer der Gewebstheorie der Wärmebildung anzusehen ist, freilich noch ohne sich über die ganze Tragweite seiner Aussagen im klaren zu sein, für die erst Felix Hoppe-Seyler (1866) Beweis und Verständnis lieferte, wurde in einer Kieler Dissertation unlängst weiter erhärtet ${ }^{55}$.

Im 19. Jahrhundert, dem der erstarkenden naturwissenschaftlichen Richtung in der Medizin setzt ein besonders intensiver Austausch ein. Die Universität Zürich, die den Mut hatte, den freisinnigen Theologen D.F.Strauss (1808-1874) $1839^{56}$ zu berufen, hat das Experiment mit dem «Materialisten» Moleschotт (1822-1893), der 1856 Heidelberg verlassen mußte, wiederholt ${ }^{57}$. Ich erwähne an deutschen Physiologen, die vorübergehend oder dauernd in der Schweiz, in der der Stern der Physiologie mit A. von Haller aufgegangen war, gelehrt haben, Carl Ludwig (1816-1895), CarL Vogt (1817-1895), Ludimar Hermann (1838-1914) ${ }^{58}$ und Hugo KronegKer (1839-1914), die zitierten Gabriel Gustav Valentin und Moritz Schiff. Auf der anderen Seite haben schweizerische Forscher, wie Albert Koelliker (1817-1905), Wilhelm His (1831-1904), der Anatom Hans Strasser (1852-1927), der Pathologe Ernst Ziegler (1849-1905) und der Psychiater Otтo Ludwig Binswanger (1852-1929), lange Jahre in Deutschland gewirkt.

Wie ist der ungewöhnlich hohe Anteil schweizerischer Forscher im Ausland zu erklären? Alphonse de Candolle, der die Besetzung der wissenschaftlichen Akademien nach Nationalitäten statistisch bearbeitet hat ${ }^{10}$, hat den Nachweis erbracht, da $\beta$ trotz beträchtlicher Fluktuationen der einzelnen Länder, die Schweiz von 1750 bis 1869 immer den ersten Platz behauptet hat: «La Suisse a maintenue sa position au haut de l'échelle.» Zweifellos gilt seine Feststellung ${ }^{59}$ : «Les Suisses ont eu de rapports inces-

${ }_{55}$ P.Schmidt-WIEDERKeHR, Theorien über Entstehungsweise und Bildungsort der Körperwärme am Ende des 18. Jahrhunderts, Diss. (Med.) Kiel 1972.

56 Vgl. Eduard Zeller, David Friedrich Strauß in seinem Leben und seinen Schriften geschildert, 2. Auflage, E.Strauß, Bonn 1874, p. 52-53.

57 Vgl. W.Moser, Der Physiologe Jakob Moleschott (1822-1893) und seine Philosophie, Diss. (Med.) Zürich 1967.

58 Von Ludimar Hermann sei auf die Zürcher Rektoratsreden Der Einflu $\beta$ der Deszendenzlehre auf die Physiologie (29. April 1878) und Die Vorbildung für das Universitätsstudium insbesondere das Medicinische (29. April 1879) hingewiesen.

59 Siehe Anm. 10, ibid., p. 199-200. 
sants et pour ainsi dire intimes avec d'autres nations. Nonseulement ils aiment les voyages, comme les Anglais, les Allemands ou les Russes, mais encore ils ont souvent résidé en pays étrangers, et là ils ont suivi des carrières libérales, sans renoncer à leur propre nationalité. De tout temps, ils ont vu se fixer chez eux des hommes instruits de toutes les nations, sans parler des simples voyageurs. On sait aussi combien d'étrangers de marque ont été élevés dans les instituts Suisses de Fellemberg, Pestalozzi, Naville etc., tandis que d'autres ont été éduqués dans leurs pays par des instituteurs suisses. Les rapports extrêmement nombreux et croisés qui résultent de cet ensemble d'usages ne peuvent s'exprimer que par le mot anglais intercourse. C'était l'effet de la petitesse du pays, de sa position géographique, de ses institutions et de l'absence d'une langue nationale.»

Die Nationen, die das Glück gehabt haben, daß der schweizerische Genius sie in ihrem Lande besuchte und beflügelte, werden sich daran mit Dankbarkeit erinnern. Das gilt in besonderem Maße für Deutschland. Und für unser Fachgebiet der Geschichte der Medizin richtet sich unser Dank an den unvergessenen Aufenthalt von Henry E. Sigerist ${ }^{60}$.

${ }^{60}$ Leiter des Sudhoff-Institutes für Geschichte der Medizin an der Universität Leipzig (1925-1932). 